\title{
Structural Variations in Prefrontal Cortex Mediate the Relationship between Early Childhood Stress and Spatial Working Memory
}

\author{
Jamie L. Hanson, ${ }^{1}$ Moo K. Chung, ${ }^{1}$ Brian B. Avants, ${ }^{2}$ Karen D. Rudolph, ${ }^{3}$ Elizabeth A. Shirtcliff, ${ }^{4}$ James C. Gee, ${ }^{1}$ \\ Richard J. Davidson, ${ }^{1}$ and Seth D. Pollak ${ }^{1}$ \\ ${ }^{1}$ Department of Psychology, University of Wisconsin-Madison, Madison, Wisconsin 53706, ${ }^{2}$ Penn Image Computing and Science Laboratory, Department \\ of Radiology, University of Pennsylvania, Philadelphia, Pennsylvania 19104, ${ }^{3}$ Department of Psychology, University of Illinois at Urbana-Champaign, \\ Champaign, Illinois 61820, and ${ }^{4}$ Department of Psychology, University of New Orleans, New Orleans, Louisiana 70148
}

\begin{abstract}
A large corpus of research indicates that exposure to stress impairs cognitive abilities, specifically executive functioning dependent on the prefrontal cortex (PFC). We collected structural MRI scans $(n=61)$, well-validated assessments of executive functioning, and detailed interviews assessing stress exposure in humans to examine whether cumulative life stress affected brain morphometry and one type of executive functioning, spatial working memory, during adolescence-a critical time of brain development and reorganization. Analysis of variations in brain structure revealed that cumulative life stress and spatial working memory were related to smaller volumes in the PFC, specifically prefrontal gray and white matter between the anterior cingulate and the frontal poles. Mediation analyses revealed that individual differences in prefrontal volumes accounted for the association between cumulative life stress and spatial working memory. These results suggest that structural changes in the PFC may serve as a mediating mechanism through which greater cumulative life stress engenders decrements in cognitive functioning.
\end{abstract}

\section{Introduction}

Stress can impact a broad range of social, cognitive, and physiological functions (Tang et al., 2006; Arnsten, 2009; Lupien et al., 2009; Schwabe et al., 2010). These effects appear to be nonlinear in nature. Exposure to low amounts of stress, for example, appears to promote beneficial effects, such as better emotional and physiological regulation (Boyce and Chesterman, 1990), enhanced cognitive functioning (Parker et al., 2005; Schwabe et al., 2010, 2011), and protective neurobiological changes, such as larger prefrontal cortices and lower cortisol levels (Lyons et al., 2002; Tang et al., 2006, 2011) after mild stress. More extreme stress has, conversely, been linked with deleterious effects on cognition, along with alterations in the hippocampus and PFC (for review, see Arnsten, 2009; Lupien et al., 2009).

A class of cognitive processes known as "executive functions" appear to be particularly vulnerable to the negative effects of stress. Executive functions encompass facets of high-order cog-

Received Jan. 20, 2012; revised March 24, 2012; accepted April 20, 2012

Author contributions: J.L.H., E.A.S., R.J.D., and S.D.P. designed research; J.L.H. and E.A.S. performed research; J.L.H., M.K.C., B.B.A., K.D.R., E.A.S., and J.C.G. contributed unpublished reagents/analytic tools; J.L.H. analyzed data; J.L.H., K.D.R., R.J.D., and S.D.P. wrote the paper.

This work was supported by National Institute of Mental Health Grants MH61285 and MH68858 (S.D.P.) and MH84051 (R.J.D.) and National Institute of Drug Abuse Fellowship DA028087 (J.L.H.). E.A.S. was at the University of Wisconsin-Madison at the time the data were collected. We thank Andrew Alexander, Michael Anderle, Patrick Bauer, Aaron Cohn, and Johnna Dorshorst for help with data collection and Andrew Fox, Brendon Nacewicz, Terrence Oakes, and Nicole Strang for helpful discussions. Lauren Allen provided thoughtful comments on a previous version of this manuscript.

Correspondence should be addressed to Jamie Hanson, Waisman Center and Department of Psychology, University of Wisconsin-Madison, 1500 Highland Avenue, Madison, WI 53705. E-mail: jlhanson5@wisc.edu.

DOI:10.1523/JNEUROSCI.0307-12.2012

Copyright $\odot 2012$ the authors $\quad 0270-6474 / 12 / 327917-09 \$ 15.00 / 0$ nition, such as inhibitory control, cognitive flexibility, working memory, and sustained attention (Zelazo et al., 2004). A large body of research in typically developing individuals, humans who have suffered brain damage, and nonhuman animal research samples has linked executive functions with the PFC (D'Esposito et al., 1995; Williams and Goldman-Rakic, 1995; Dias et al., 1996; Owen et al., 1996). However, little is currently known about the neurobiological correlates of stress-induced changes in executive functions in the developing organism.

Chronic levels of high stress lead to structural changes of the PFC in rodents, with reduced dendritic arborization and lower spine density (Cook and Wellman, 2004; Liston et al., 2006; Radley et al., 2006; Holmes and Wellman, 2009). Such chronic stress has also been linked with executive function deficits in rodents, nonhuman primates, and humans (Murphy et al., 1996; Sanchez et al., 1998; Oei et al., 2006; Evans and Schamberg, 2009; Holmes and Wellman, 2009). This previous research has been conducted in adult humans and nonhuman animals; it is not clear whether these patterns would be seen early in development.

During childhood and adolescence, the PFC has a protracted course of brain development with alterations seen until the second decade of life (Lenroot and Giedd, 2006), making it potentially vulnerable to the effects of chronic early-life stress. Chronic stress, especially very high levels, may affect cognitive functions linked to this region. This study examined whether individual differences in the PFC mediated the effects of cumulative life stress on executive functioning during development.

To probe these questions, we used detailed interview metrics of cumulative life stress, well-validated assessments of executive functioning, and neuroimaging methodology appropriate for use 
Table 1. Demographic information

\begin{tabular}{lcccc}
\hline & Sull subjects & $\begin{array}{l}\text { Subjects with MRI scans, } \\
\text { YLSI, and CANTAB data }\end{array}$ & $\begin{array}{c}\text { Subjects with only } \\
\text { MRI scans and YLSI data }\end{array}$ & $\begin{array}{c}p \text { values (comparing subjects } \\
\text { with and without CANTAB data) }\end{array}$ \\
\hline Age (mean \pm SD months) & $142.357 \pm 21.1226$ months & $140.714 \pm 19.758$ months & $146.610 \pm 24.44$ months & $F=0.954, p=0.333$ \\
Puberty (Tanner staging) & $1.725 \pm 1.283$ & $1.7045 \pm 1.280$ & $1.7794 \pm 1.328$ & $F=0.041, p=0.840$ \\
Whole-brain volume (mm ${ }^{3}$ ) & $1431.792 \pm 130.30$ & $1431.202 \pm 117.34$ & $1433.3212 \pm 163.30$ & $F=0.003, p=0.955$ \\
Gender (male, female) & 32 males, 29 females & 22 males, 22 females & 10 males, 7 females & $\chi^{2}=0.383, p=0.536$ \\
SES (as indexed by maternal education) & $6.0851 \pm 0.974$ & $6.20 \pm 0.868$ & $5.75 \pm 1.215$ & $F=1.946, p=0.170$ \\
\hline
\end{tabular}

Maternal education varied on a numeric scale from 1 to 8 , denoting level of education obtained with possible choices of grade school, high school or general education diploma, 2-year college, trade, or technical school, 4 -year college, or graduate school. SES, Socioeconomic status.

with children. Using these tools, we aimed to further elucidate whether (1) cumulative life stress affected working memory (one executive function) and (2) the extent to which individual differences in PFC morphometry mediated this effect.

\section{Materials and Methods}

Participants. Sixty-one children (32 males, 29 females; mean age, $142.35 \pm 21.12$ months) completed the Youth Life Stress Interview (YLSI) and MRI scanning (for additional demographic information, see Table 1). Maternal education was used as an index of socioeconomic status in this study because this measure is strongly associated with child health, household income, and stimulation in the environment (Haveman and Wolfe, 1995; Waldfogel et al., 2002). Children were recruited from the Madison, Wisconsin community by posting fliers. Participants were given $\$ 60$ for a 4 hour visit to the laboratory. Such recruitment procedures were similar to the large body of normative pediatric brain imaging studies (Asato et al., 2010; Guyer et al., 2012).

To rule out physical abuse or other forms of child maltreatment, parents completed the Parent-Child Conflict Tactics Scale (PC-CTS) (Straus et al., 1998), and local Department of Human Services records were examined. The PC-CTS is a 20 -item measure of the frequency with which a parent has performed specific acts of physical aggression toward the child. Parents who scored $\geq 20$ on the physical abuse subscale on the PC-CTS and/or had substantiated cases of physical abuse on record with the Dane County Department of Human Services were excluded from all analyses. Maltreatment was specifically ruled out because of potential unique alteration in brain and behavior stemming from this early adversity (Hanson et al., 2010).

Measurements of puberty. Cumulative life stress may affect pubertal maturation (Ellis, 2004), which may have direct or indirect influences on brain development. To control for the influence of puberty, pediatric nurse practitioners completed Tanner staging on the children using a physical exam (Shirtcliff et al., 2009). This involved brief breast palpation for girls and visual inspection of breast and pubic hair development. An orchidometer was used to measure testicular size in boys, along with visual inspection of genitals and pubic hair. Puberty scores ranged from 1 (no development) to 5 (adult development).

Executive functioning assessment. To examine executive functioning, we used the spatial working memory (SWM) subtest of the Cambridge Neuropsychological Test Automated Battery (CANTAB) (Cambridge Cognition). The CANTAB has been used extensively with children and adolescents (Luciana and Nelson, 2002) and has proved sensitive in discriminating various clinical populations from typically developing children. The CANTAB is computerized for standardized administration. The stimuli cannot be verbalized and the subtasks require nonverbal responses; thus, performance is not confounded with subjects' verbal skills. CANTAB data for the SWM subtest was available for 44 participants (Table 1). The SWM subtest involves touching boxes on a screen to find a token. Participants must, through a process of elimination, find one blue "token" in one of the boxes presented. This portion of the CANTAB reflects an individual's visuospatial working memory. A participant's score is based on total errors (touching boxes that have already been found to be empty and revisiting boxes that have already been revealed to contain a token). Raw performance data are $Z$-transformed based on age-based neurocognitive norms for each subject's age and gender. Higher $Z$-scores indicate better performance on this task and a lower number of total errors.

Assessment of cumulative life stress. To assess cumulative life stress, interviewers administered the lifetime adversity section of the YLSI (Rudolph and Hammen, 1999; Rudolph and Flynn, 2007) separately to children and their parents. Postdoctoral fellows, advanced graduate students, and postbaccalaureate staff conducted all interviews. Interviewers received intensive training and ongoing consultation from the developers of the YLSI at the University of Illinois at Urbana-Champaign. This interview assessed children's exposure to severe negative life events and circumstances across their lifetime, excluding events within 1 year to distinguish recent life stressors. General and specific probes were used to assess a child's exposure to particularly stressful events and circumstances (e.g., death of a close family member or friend, exposure to severe marital conflict, and severe chronic illness of a close family member or friend). Semi-structured follow-up questions were then asked to assess the context of the event (e.g., timing, duration, objective consequences).

Integrating across parent and child reports, an independent rating team of three to seven members provided a consensual rating on a 10 point scale that reflected the overall level of cumulative life stress. This coding of the YLSI incorporated a detailed consideration of the context of events and the impact on an individual child's life rather than simply reflecting the sum of the number of stressors. For example, a parent's loss of employment receives a uniform score within many stress checklist approaches, but the YLSI differentiates a parent leaving an unsatisfying job because of lack of financial need from being fired after numerous years of fulfilling and dedicated service (Wethington et al., 1995). To detail specific examples from our study, a score of a 1 was given to a child whose pet was hit by a car but was not seriously injured. A score of a 5 was given to a child who was in foster care early in life, had multiple moves, and also had one of their parents die early in life. A score of a 7.5 was given to a child whose parent had serious chronic medical issues that also caused instability in employment. Also reflected in this score were the facts that this child's sibling had serious medical and mental health issues, there was serious marital conflict in the family resulting in parental separation, and one of the child's parents was incarcerated extensively. A score of a 10 was given to a child who was homeless, had several close family members die unexpectedly, and whose parents had a highly conflicted relationship that resulted in separation. A key point is that the scores not only reflect the objective stressors but also the subjective impact of these events as perceived by the child. This rating system has high reliability and validity (Rudolph and Flynn, 2007) (intraclass correlation coefficient of 0.99).

Assessment of secondary psychopathology. To assess child psychopathology, parents completed the computerized Diagnostic Inventory for Children and Adolescents (Reich, 2000) regarding their child. Responses were used to generate specific Diagnostic and Statistical Manual of Mental Disorders diagnoses. Three children were diagnosed with Attention Deficit Hyperactivity Disorder; our results are not changed by removal of these subjects.

MRI acquisition. All children completed one MRI scan. Highresolution anatomical MRI images were obtained using a 3 T GE SIGNA (GE Healthcare) scanner with a quadrature radio frequency head coil. A three-dimensional, inversion recovery pulse sequence was used to gen- 
erate T1-weighted images with the following parameters: TR, $21 \mathrm{~ms}$; TE, $8 \mathrm{~ms}$; flip angle, $308^{\circ} ; 240 \mathrm{~mm}$ field of view; $256 \times 192$ in-plane acquisition matrix (interpolated on the scanner to $256 \times 256$ ); and 128 axial slices (1.2 mm thick) covering the whole brain. Before MRI scanning, participants were oriented to the MRI through the use of a mock-MRI simulator. During MRI acquisition, participants were instructed to stay as still as possible and were able to watch a movie of their choosing.

Diffeomorphic image normalization, template creation, and tensor-based morphometry. T1-weighted images were corrected for field inhomogeneity and masked to exclude all extraneous aspects of the brain (e.g., dura matter, skull). These masked images were then used in template creation. After going through a six-parameter rigid-body transformation, each individual brain was registered to our template using symmetric normalization (SyN). This algorithm allows for large deformations but also constrains the deformations to be physically reasonable. The nonlinear transformations resulting from the SyN algorithm also provide deformation tensor fields, defined in the optimal template space, that describe the voxelwise shape change from the template to each subject's brain. Jacobian determinants of the deformation field indicate the fractional volume expansion and contraction at each voxel, quantifying the magnitude of regional volume alterations required to match the template. Before the statistical testing, this adjusted Jacobian map was subjected to a log transformation to make the distribution closer to the normal distribution (Avants and Gee, 2004). Jacobian determinants were then smoothed with a $4 \mathrm{~mm}$ full-width, half-maximum Gaussian filter.

The MRI template was study specific, constructed based on all subjects. Template construction consisted of a multi-resolution strategy (for this study, a four-level Gaussian pyramid) as well as the similarity metric for the optimization, along with a maximum number of iterations. We used the region-based cross-correlation similarity metric, which is optimal in dealing with locally varying inhomogeneity in the appearance of images. The maximum number of iterations in the normalization was set to 200 , although convergence may have occurred before the maximum was reached. This approach is particularly applicable to pediatric populations, because it minimized sources of variability (e.g., brain tissue segmentation), used a study-specific anatomical template, and yielded high sensitivity at the voxel level. In addition, recent research validated SyN as one of the best available warping algorithms in a recent comparison of 14 nonlinear registration algorithms (Klein et al., 2009).

\section{Results}

\section{Descriptive statistics for life stress and relationship with other variables}

The mean rating of cumulative life stress was 3.14 \pm 2.009 (of 10). The correlation between cumulative life stress and age was nonsignificant $(r=0.157, p=0.226)$, whereas puberty and cumulative life stress were significantly correlated $(r=0.263, p=0.04)$. This finding argues against stress exposure being a simple facet of age and more related to life experiences but underscores that life stress may be affecting pubertal maturation.

\section{Test of formal mediation}

Hierarchical multiple regression analyses were conducted to specifically examine whether individual differences in the PFC mediated the association between cumulative life stress and executive function. A variable is thought to be mediator when it carries the influence of a given independent variable (IV) to a given dependent variable (DV). A formal test of mediation involves establishing numerous criteria (Baron and Kenny, 1986), each of which is detailed below.

\section{Relationship between cumulative life stress and PFC structure and function (criterion 1)}

Criterion 1 requires that the IV (cumulative life stress) is significantly associated with the mediator (the prefrontal regions of interest). We established criterion 1 based on a logical AND con-
Table 2. Negative associations between cumulative life stress and brain structure (greater cumulative life stress was related to smaller volumes)

\begin{tabular}{llll}
\hline Region & $\begin{array}{l}\text { Cluster size; } \\
\text { corrected } p \text { value }\end{array}$ & $\begin{array}{l}\text { Pearson's } \\
r \text { value }\end{array}$ & $\begin{array}{l}\text { Cluster coordinates } \\
\text { (MNI space) }\end{array}$ \\
\hline Right putamen & 249 voxels; $p<0.001$ & $r=-0.525$ & $+28,+7,+0$ \\
Left putamen & 62 voxels; $p=0.016$ & $r=-0.377$ & $-21,+11,+1$ \\
Right occipital lobe & 233 voxels; $p<0.001$ & $r=-0.419$ & $+30,-88,-13$ \\
Right occipital lobe & 72 voxels; $p=0.007$ & $r=-0.439$ & $+9,-88,-4$ \\
Left occipital lobe & 65 voxels; $p=0.013$ & $r=-0.456$ & $-8,-96,-18$ \\
Right middle temporal gyrus & 175 voxels; $p<0.001$ & $r=-0.418$ & $+52,-11,-12$ \\
Left middle temporal gyrus & 151 voxels; $p<0.001$ & $r=-0.469$ & $-62,-17,-7$ \\
Right parietal white matter & 109 voxels; $p<0.001$ & $r=-0.429$ & $+32,-27,+33$ \\
Right middle parietal gyrus & 67 voxels; $p=0.011$ & $r=-0.411$ & $+59,-42,+9$ \\
Right precuneus & 137 voxels; $p=0.001$ & $r=-0.438$ & $+8,-58,+14$ \\
Right cerebellum & 137 voxels; $p<0.001$ & $r=-0.415$ & $+41,-67,-22$ \\
Ventral medial PFC & 189 voxels; $p<0.001$ & $r=-0.418$ & $+0,+23,-24$ \\
Right prefrontal white matter & 121 voxels; $p<0.001$ & $r=-0.417$ & $+18,+38,+1$ \\
Right frontal white matter & 87 voxels; $p=0.002$ & $r=-0.461$ & $+18,-12,+42$ \\
Left frontal pole & 98 voxels; $p<0.001$ & $r=-0.422$ & $-20,+62,-9$ \\
Left anterior cingulate & 50 voxels; $p=0.043$ & $r=-0.424$ & $-11,+45,+17$ \\
\hline
\end{tabular}

No positive associations with cumulative life stress and brain structure survived correction for multiple comparisons. All regions noted significant at $p<0.05$ corrected for multiple comparisons, initial statistical threshold $p=0.000$ uncorrected.

junction analysis. Such an analytic approach allowed first for the isolation of regions related to stress and then to examine correlations between volumetric properties of these specific regions and working memory. To do this, we used the voxelwise correlations of Jacobian determinants (a metric of volumetric expansion or contraction) and the child's cumulative life stress score. This correlation was conducted in FMRISTAT (Worsley et al., 2002) and combined with voxelwise correlations of Jacobian determinants with working memory performance $Z$-scores, a prefrontally mediated function. In these analyses, whole-brain volume and pubertal status were covaried. A statistical threshold of $t_{(58)}=3.966$, $p=0.05$ corrected was used for cumulative life stress scores (Table 2), and a threshold of $t_{(41)}=2.967, p=0.005$ uncorrected was used for working memory subscores. These correlations were then used in a logical AND conjunction analysis to identify the brain regions that were associated with both cumulative life stress and executive functioning. Assuming independence of this test, these results are significant at $0.000025(0.005 \times 0.005)$, uncorrected. To generate coefficients for use in the mediation analysis for this criterion, we ran multiple regression analyses with cumulative life stress entered as the IV and the prefrontal regions of interest that emerged from our voxelwise analyses entered as DVs in separate regression models controlling for variations in pubertal status (Table 3).

As hypothesized, portions of the PFC were smaller with elevated cumulative life stress, and smaller PFC volumes were associated with poorer executive functioning. These associations emerged as two clusters in the PFC: one located in gray matter near the anterior cingulate and the frontal poles and one located in white matter near the forceps minor (Figs. 1,2). This analysis isolates brain regions of interest that are linked with both cumulative life stress and SWM (specifically, the total number of errors on the SWM subtest; Table 4). In these and all analyses, a $Z$-score of SWM errors was used; a higher $Z$-score indicated fewer errors on this neurocognitive task. The peak coordinates for all analyses were mapped to MNI space by registering the custom template to the MNI152 Average Template (Montreal Neurological Institute) using the SyN algorithm with similar settings to our subject-level normalization (e.g., crosscorrelation similarity metric; maximum of 200 iterations). 
Table 3. Criterion 1 for prefrontal regions of interest: IV (cumulative life stress) is significantly associated with the mediator (the prefrontal regions of interest)

\begin{tabular}{|c|c|c|c|c|}
\hline$\overline{D V}$ & IV & Unstandardized regression coefficients, $\mathrm{SE}, p$ value & $F \Delta_{(\mathrm{df})}$ & $R^{2}$ change \\
\hline Frontal gray matter (left anterior cingulate cluster) & YLSI & $\mathrm{B}=-0.059, \mathrm{SE}=0.016, p=0.001$ & $F \Delta_{(1,57)}=13.2$ & $R^{2} \Delta=0.173$ \\
\hline Frontal white matter (right prefrontal white matter cluster) & YLSI & $\mathrm{B}=-0.031, \mathrm{SE}=0.008, p=0.001$ & $F \Delta_{(1,57)}^{(1, J)}=13.6$ & $R^{2} \Delta=0.138$ \\
\hline
\end{tabular}

\section{Association between cumulative life stress and executive functioning (criterion 2)}

Criterion 2 requires that the IV is significantly associated with the DV (working memory performance) in the absence of the mediator. To establish criteria 2, we conducted multiple regression analyses in which cumulative life stress was entered as the IV and working memory performance scores were entered as DVs in separate regression models. Pubertal status was entered at step 1 to adjust for any possible association between puberty and memory performance.

Providing support for criterion 2, heightened cumulative life stress was associated with poor executive functioning, as indexed by memory errors $(r=-0.474$, $p=0.001)$. Multiple regression analysis revealed that this association was significant after controlling for individual differences in pubertal status $\left(F \Delta_{(1,41)}=11.56 ; R^{2} \Delta=\right.$ $0.219 ; p=0.002)($ Table 5$)$.

\section{Test of formal mediation (criterion 3 )}

Criterion 3 requires that the mediator has a significant unique effect on the DV after adjusting for the IV, and the effect of the IV on the DV is reduced on the addition of the mediator to the model. To establish criteria 3 , we conducted multiple regression analyses in which cumulative life stress was entered at step 1 (while adjusting for pubertal status) and the brain regions resulting from the logical AND conjunction analysis corrected for wholebrain volume were entered at step 2 (Table 5). In these models, we entered the log-Jacobian determinant for each participant from the prefrontal cluster identified by the logical AND conjunction analysis (of the whole-brain correlation with cumulative life stress and the whole-brain correlation with executive functioning; see below). The output of this model contains regression coefficients and measurement of SE for the effect of both cumulative life stress and prefrontal morphometry on executive functioning.

As shown in Table 5, the necessary conditions for mediation were met because the IV affected the mediator, the mediator had a significant unique effect on the DV, and the effect of the IV on the DV lessened after the addition of the mediator to the model. When the prefrontal

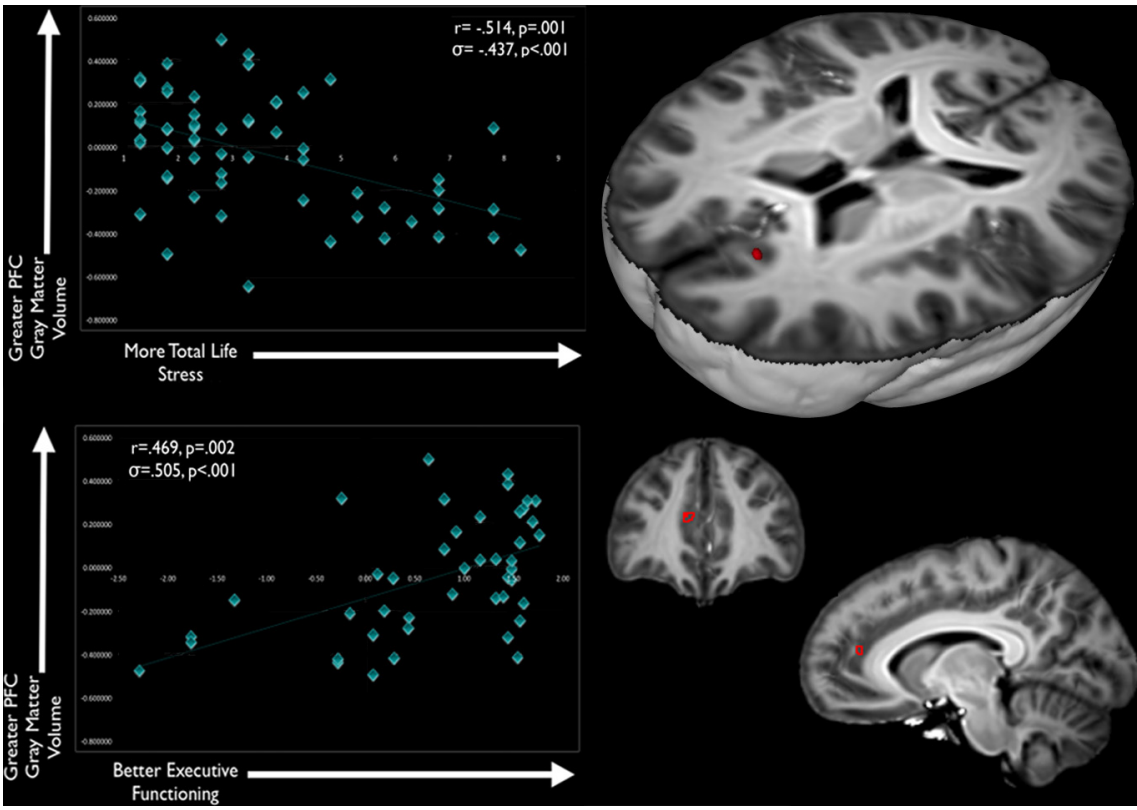

Figure 1. Variations in PFC gray matter are associated with individual differences in both cumulative life stress and SWM performance. Brain images show the results of logical AND conjunction analyses, in which PFC gray matter is negatively associated with cumulative life stress ( $p<0.005$, uncorrected) and positively associated with SWM performance (as indexed by fewer errors and higher Z-scores) $(p<0.005$, uncorrected). The scatter plot for cumulative life stress and PFC gray matter volume is shown in the top left corner, whereas the scatter plot for SWM performance and PFC gray matter volume is shown in the bottom left corner.

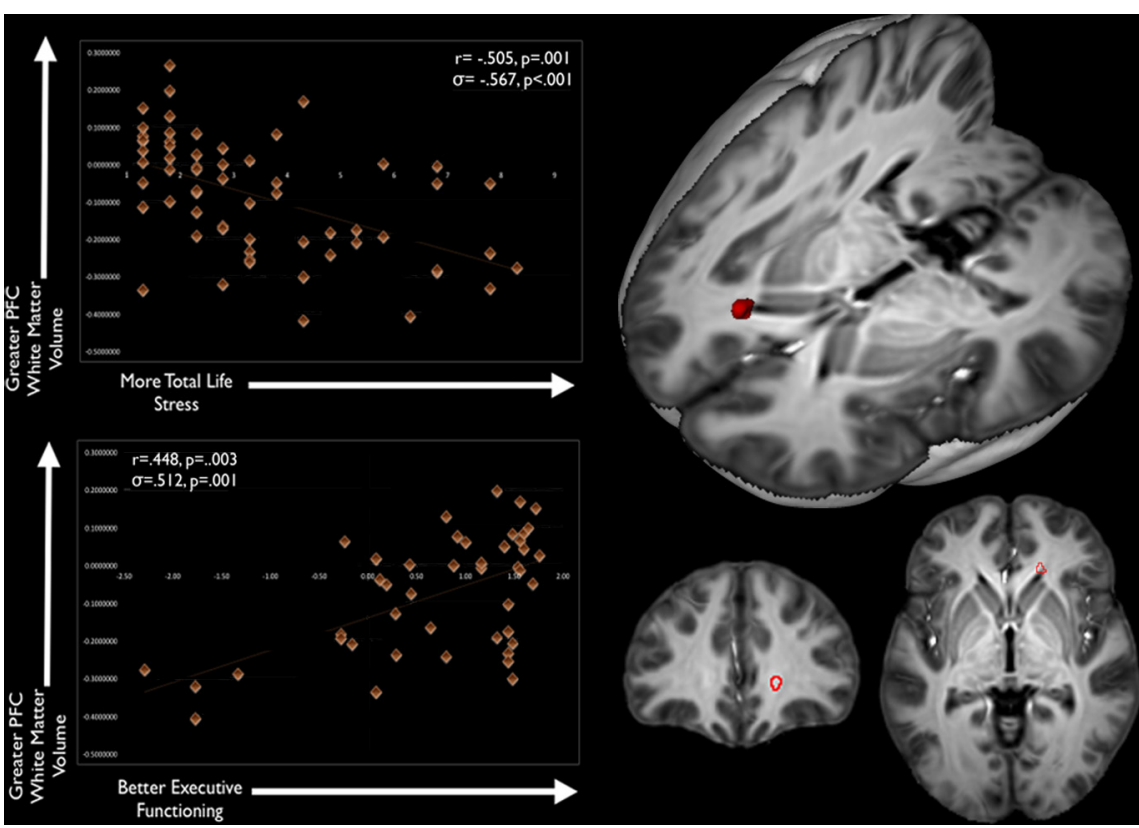

Figure 2. Variations in PFC white matter are associated with individual differences in both cumulative life stress and SWM performance. Brain images show the results of logical AND conjunction analyses, in which PFC white matter is negatively associated with cumulative life stress $(p<0.005$, uncorrected) and positively associated with SWM performance (as indexed by fewer errors and higher $Z$-scores) $(p<0.005$, uncorrected). The scatter plot for cumulative life stress and PFC white matter volume is shown in the top left corner, whereas the scatter plot for SWM performance and PFC white matter volume is shown in the bottom left corner. 
Table 4. Clusters in the PFC related to both cumulative life stress and SWM

\begin{tabular}{|c|c|c|c|c|c|}
\hline Cluster size (size in $1 \mathrm{~mm}^{3}$ ) & $\begin{array}{l}\text { Pearson's correlation } \\
\text { with YLSI, } p \text { value }\end{array}$ & $\begin{array}{l}\text { Spearman's correlation } \\
\text { with YLSI, } p \text { value }\end{array}$ & $\begin{array}{l}\text { CANTAB Z-score, } \\
\text { Pearson's correlation, } p \text { value }\end{array}$ & $\begin{array}{l}\text { CANTAB Z-score, Spearman's } \\
\text { correlation, } p \text { value }\end{array}$ & $\begin{array}{l}\text { Approximate MNI } \\
\text { coordinates }\end{array}$ \\
\hline 49 voxels & $r=-0.505, p=0.001$ & $r_{s}=-0.567, p<0.001$ & Total errors: $r=0.448, p=0.003$ & Total errors: $r_{s}=0.512 ; p<0.001$ & $+18,+36,0$ \\
\hline 13 voxels & $r=-0.514, p=0.001$ & $r_{s}=-0.437, p<0.001$ & Total errors: $r=0.469, p=0.002$ & Total errors: $r_{s}=0.505 ; p<0.001$ & $-11,+45,+15$ \\
\hline
\end{tabular}

All results are partial correlations, controlling for individual difference in whole-brain volume and pubertal status.

Table 5. Criteria 2 and 3 for SWM total errors Z-scores, cumulative life stress, and prefrontal regions of interest [DV was SWM total errors Z-scores (higher Z-score $=$ fewer errors)]

\begin{tabular}{|c|c|c|c|}
\hline Variables & Unstandardized regression coefficients, $\mathrm{SE}, p$ value & $F \Delta_{(\mathrm{df})}$ & $R^{2}$ change, $p$ values \\
\hline \multicolumn{4}{|l|}{ Model 1} \\
\hline Pubertal status & $B=0.009, \mathrm{SE}=0.112, p=0.937$ & $F \Delta_{(2,41)}=5.933$ & $R^{2} \Delta=0.224, p=0.005$ \\
\hline Cumulative life stress & $B=-0.245, \mathrm{SE}=0.072, p=0.002$ & & \\
\hline \multicolumn{4}{|l|}{ Model 2} \\
\hline Pubertal status & $B=0.072, \mathrm{SE}=0.101, p=0.481$ & $F \Delta_{(2,39)}=8.037$ & $R^{2} \Delta=0.226, p=0.001$ \\
\hline Cumulative life stress & $B=-0.038, \mathrm{SE}=0.082, p=0.647$ & & \\
\hline Frontal gray matter (left anterior cingulate cluster) & $B=1.37, \mathrm{SE}=0.532, p=0.014$ & & \\
\hline Frontal white matter (right prefrontal white matter cluster) & $B=2.834, \mathrm{SE}=0.925, p=0.004$ & & \\
\hline
\end{tabular}

Criterion 2 requires that the IV (cumulative life stress) is significantly associated with the DV (SWM performance) in the absence of the mediator. Criterion 3 requires that the mediator (prefrontal regions of interest) has a significant unique effect on the DV after adjusting for the IV, and the effect of the IV on the DV is reduced after the addition of the mediator to the model.

Table 6. Brain regions not hypothesized that were also related to both cumulative life stress and executive functioning

\begin{tabular}{lllll}
\hline Region & Cluster size $\left(\right.$ size in $\left.1 \mathrm{~mm}^{3}\right)$ & Correlation with YLSI, $p$ value & CANTAB Z-score, correlation, $p$ value & Approximate MNI coordinates \\
\hline Temporal lobe (near middle temporal gyrus) & 41 voxels & $r=-0.346, p=0.025$ & Total errors: $r=0.560, p<0.001$ & $(+57,-6,-19)$ \\
Precuneus & 21 voxels & $r=-0.381, p=0.013$ & Total errors: $r=0.457, p=0.002$ & $(+52,+8,-34)$ \\
\hline
\end{tabular}

All results are partial correlations, controlling for individual difference in whole-brain volume and pubertal status.

regions of interests were added to the model, the association between cumulative life stress and executive functioning was nonsignificant for total errors. Of important note, variations in the PFC significantly predict executive functioning even after accounting for stress (as indexed by an association between prefrontal clusters and SWM when cumulative life stress is included in our simultaneous regression models).

To further establish mediation (Preacher and Hayes, 2004), we conducted Sobel tests (Sobel, 1986) using an interactive Sobel test calculator to examine the significance of the indirect effects of cumulative life stress on executive functioning as mediated by prefrontal volumes. These tests revealed that frontal white matter volume $(Z=-2.11, \mathrm{SE}=0.038, p=0.034)$ and frontal gray matter volume $(Z=-2.31, \mathrm{SE}=0.038, p=0.02)$ mediated the association between life stress and SWM total errors $Z$-score, a metric of SWM performance.

Similar results were seen when controlling for age. All of the criteria detailed previously were met. In addition, frontal white matter volume $(Z=-1.98, \mathrm{SE}=0.035, p=0.047)$ and frontal gray matter volume $(Z=-2.190, \mathrm{SE}=0.037, p=0.028)$ mediated the association between cumulative life stress and SWM total errors $Z$-score.

Relationships between other brain structures, cumulative life stress, and CANTAB performance

In addition to hypothesized differences in the PFC, examination of the logical AND conjunction analysis revealed that parts of the temporal lobe and the precuneus were associated with both cumulative life stress and executive functioning (Table 6). Greater cumulative life stress was associated with smaller volumes in these regions, and smaller volumes in these regions were associated with poorer executive functioning.

\section{Effects of acute versus chronic stress}

As noted previously, we assessed different facets of life stress (i.e., cumulative life stress or acute stress in the last year) through use of the YLSI. To interrogate the unique effects of acute stress, the average of parent's and child's reports of stress in the past year was entered into regression models as an IV along with pubertal stage, whole-brain volume, cumulative life stress, and prefrontal regions of interest. SWM performance (as indexed by total errors) was then entered as the DV. Interestingly, as shown in Table 7, the effects of stress on SWM are more strongly related cumulative life stress rather than stressors in the past year (lifetime stress, $p=0.030$; acute stress, $p=0.363$ ). Of important note, our brain regions of interest are still significant when acute stress is present in our regression models (frontal white matter, $p=0.038$; frontal gray matter, BA $10, p=0.028$ ).

\section{Additional examination of brain regions affected by stress}

As detailed in Table 2, a number of brain regions were related to cumulative life stress. Our analyses are conducted on a voxelwise basis to more specifically localize the effects of stress. Multiple independent clusters of interest merged in specific regions (e.g., two right occipital clusters). Each of these clusters uniquely contributes to associations between stress exposure and alterations in the brain, so they are detailed here. These relationships were significant using both Pearson's and Spearman's correlations, indicating that such results were not driven by outliers.

We probed whether these same regions were related to behavior through the use of logical AND conjunction analyses in which we looked for spatial overlap between voxelwise correlations with cumulative life stress and voxelwise correlations with SWM. The majority of the regions related to cumulative life stress were not related to behavior, because only two clusters in the PFC along with clusters in the precuneus and middle temporal gyrus emerged from voxelwise correlations analyses. Such analyses may be under-powered so we also examined basic bivariate correlations for brain regions that emerged from voxelwise correlations with cumulative life stress. These analyses yielded similar results, because only portions of prefrontal gray matter (near the left anterior cingulate), prefrontal white matter, right middle tempo- 
Table 7. Regression-dependent variable: SWM performance (as indexed by total error Z-score; higher Z-score = fewer errors)

\begin{tabular}{|c|c|c|c|}
\hline Variables & Unstandardized regression coefficients, SE, $p$ value & $F \Delta_{(\mathrm{df})}$ & $R^{2}$ change, $p$ values \\
\hline \multicolumn{4}{|l|}{ Model 1} \\
\hline Pubertal stage & $B=0.113, \mathrm{SE}=0.109, p=0.308$ & \multirow[t]{2}{*}{$F \Delta_{(1,41)}=7.477$} & \multirow[t]{2}{*}{$R^{2} \Delta=0.359, p<0.001$} \\
\hline Cumulative life stress & $B=-0.205, \mathrm{SE}=0.068, p=0.001$ & & \\
\hline \multicolumn{4}{|l|}{ Model 2} \\
\hline Pubertal stage & $B=0.134, \mathrm{SE}=0.112, p=0.238$ & \multirow{3}{*}{$F \Delta_{(1,39)}=0.847$} & \multirow{3}{*}{$R^{2} \Delta=0.014, p=0.363$} \\
\hline Cumulative life stress & $B=-0.172, \mathrm{SE}=0.077, p=0.030$ & & \\
\hline Acute stress & $B=-0.35, \mathrm{SE}=0.038, p=0.363$ & & \\
\hline \multicolumn{4}{|l|}{ Model 3} \\
\hline Pubertal stage & $B=0.092, \mathrm{SE}=0.104, p=0.380$ & \multirow{5}{*}{$F \Delta_{(2,37)}=4.671$} & \multirow[t]{5}{*}{$R^{2} \Delta=0.126, p=0.016$} \\
\hline Cumulative life stress & $B=-0.010, \mathrm{SE}=0.088, p=0.908$ & & \\
\hline Acute stress & $B=-0.033, \mathrm{SE}=0.035, p=0.352$ & & \\
\hline Frontal white matter (right prefrontal white matter cluster) & $B=2.142, \mathrm{SE}=0.995, p=0.038$ & & \\
\hline Frontal gray matter (left anterior cingulate cluster) & $B=1.213, \mathrm{SE}=0.530, p=0.028$ & & \\
\hline
\end{tabular}

Table 8. Regression DV: SWM performance (as indexed by total error Z-score; higher Z-score $=$ fewer errors)

\begin{tabular}{ll}
\hline Variables & $\begin{array}{l}\text { Unstandardized regression } \\
\text { coefficients, SE, } p \text { value }\end{array}$ \\
\hline Pubertal stage & $B=0.1, \mathrm{SE}=0.097, p=0.308$ \\
Right middle temporal gyrus & $B=0.720, \mathrm{SE}=0.732, p=0.332$ \\
Right parietal white matter & $B=0.440, \mathrm{SE}=1.03, p=0.671$ \\
$\begin{array}{l}\text { Prefrontal gray matter (near the left anterior } \quad \text { cingulate) } \\
\text { Right prefrontal white matter }\end{array}$ & $B=1.410, \mathrm{SE}=0.490, p=0.007$ \\
\hline
\end{tabular}

ral gyrus, and right parietal white matter were related to SWM at $p<0.05$. These regions were then entered into a multiple regression model with the brain regions entered as IVs and SWM was entered as the DV. Only portions of the PFC (both gray and white matter clusters) were associated with SWM at $p<0.05$ as shown in Table 8.

\section{Multiple regression analyses with $\mathrm{PFC}$ and other regions from} conjunction analyses

To probe the specificity of our results, we conducted multiple regression analyses with all of the regions that emerged from the logical AND conjunction analyses. These regions were associated with both cumulative life stress and behavior (SWM errors). Total SWM errors was entered as the DV, whereas cumulative life stress, pubertal stage, acute stressors that occurred in the past year, and the four brain clusters of interest (clusters in prefrontal white matter, prefrontal gray matter, precuneus, and a portion of the medial temporal lobe) were entered as IVs. The clusters in the PFC were the only variables that are significant at $p<0.05$ in simultaneous regression models (Table 9). There was a nearsignificant association with the precuneus $(p=0.053)$ and SWM errors.

\section{Asymmetrical effects of cumulative life stress on the brain}

We conducted exploratory analyses to assess asymmetry in the relationships between cumulative life stress and brain morphometry, because many effects were lateralized. We used one-way ANOVAs to compare clusters identified previously (Table 2) against inverted versions of these same clusters (flipped left to right hemisphere, or right to left). We found significant asymmetrical effects for the left frontal pole $(p<0.001)$, along with portions of the right $(p<0.001)$ and left $(p=0.009)$ occipital lobe. A trend toward asymmetrical effects was also noted for the putamen. Means, SD, and $p$ values for this preliminary test of asymmetry are shown in Table 10.
Table 9. Regression DV: SWM performance (as indexed by total error Z-score; higher Z-score $=$ fewer errors)

\begin{tabular}{|c|c|}
\hline Variables & $\begin{array}{l}\text { Unstandardized regression } \\
\text { coefficients, SE, } p \text { value }\end{array}$ \\
\hline \multicolumn{2}{|l|}{ Model 1} \\
\hline Cumulative life stress & $B=-0.244, \mathrm{SE}=0.07, p=0.001$ \\
\hline \multicolumn{2}{|l|}{ Model 2} \\
\hline Cumulative life stress & $B=0.038, \mathrm{SE}=0.085, p=0.661$ \\
\hline Acute stress & $B=-0.039, \mathrm{SE}=0.035, p=0.269$ \\
\hline Temporal lobe (near middle temporal gyrus) & $B=0.841, \mathrm{SE}=0.813, p=0.308$ \\
\hline Precuneus cluster & $B=1.335, \mathrm{SE}=0.666, p=0.053$ \\
\hline $\begin{array}{l}\text { Frontal white matter (right prefrontal white } \\
\text { matter cluster) }\end{array}$ & $B=2.020, \mathrm{SE}=0.988, p=0.049$ \\
\hline $\begin{array}{l}\text { Frontal gray matter (left anterior cingulate } \\
\text { cluster) }\end{array}$ & $B=1.065, \mathrm{SE}=0.5, p=0.04$ \\
\hline
\end{tabular}

Whole-brain volume has been controlled for with all brain regions of interest.

\section{Discussion}

Our findings indicate that cumulative life stress is associated with structural variations in the PFC, such that heightened cumulative life stress is associated with smaller volumes in both white and gray matter. Both cumulative life stress and smaller PFC volumes are associated with individual differences in an important domain of executive functioning SWM. Higher levels of cumulative life stress were associated with poorer SWM performance, whereas smaller PFC volumes (in both gray and white matter) were associated with poorer SWM.

These data are consistent with research showing that stress can impair cognitive functioning, as well as with research in human and nonhuman animals showing stress-specific alterations in the PFC (for review, see Arnsten, 2009). Previous pediatric and adult imaging research has found an association between greater white matter volumes and superior performance in various cognitive tasks (Sowell et al., 2001; Brickman et al., 2006). For gray matter, our research finding fits with adult imaging studies showing less gray matter being related to lower performance on cognitive tasks (Gunning-Dixon and Raz, 2003; Tisserand et al., 2004). However, contextualizing these results in an adolescent sample in which the brain is still developing rapidly is more complex. Adolescent subjects who have experienced a great deal of stress may be "losing" gray matter at a greater rate (in addition to the normative reductions in gray matter seen in development, reported by others; Lenroot and Giedd, 2006) than subjects who have experienced less stress. It is possible that stress-induced impairments in cognitive functioning may have similar neurobiological correlates across the lifespan, with lower gray and white matter 
Table 10. Asymmetrical effects of cumulative life stress on the brain

\begin{tabular}{|c|c|c|c|c|}
\hline & Original region (MNI coordinates) & Mean $\pm S D$ of original cluster & Mean $\pm S D$ of inverted cluster & Test of the difference between regions \\
\hline Right putamen & $+28,+7,+0$ & $-0.136 \pm 0.132$ & $-0.092 \pm 0.122$ & $F=3.675, p=0.056$ \\
\hline Left putamen & $-21,+11,+10$ & $-0.161 \pm 0.123$ & $-0.149 \pm 0.127$ & $F=0.259, p=0.612$ \\
\hline Right occipital lobe & $+30,-88,-13$ & $0.076 \pm 0.253$ & $0.057 \pm 0.224$ & $F=0.191, p=0.662$ \\
\hline Right occipital lobe & $+9,-88,-4$ & $0.017 \pm 0.236$ & $-0.158 \pm 0.264$ & $F=14.875, p<0.001$ \\
\hline Left occipital lobe & $-8,-96,-18$ & $0.217 \pm 0.177$ & $0.136 \pm 0.158$ & $F=7.087, p=0.009$ \\
\hline Right middle temporal gyrus & $+52,-11,-12$ & $-0.102 \pm 0.218$ & $-0.127 \pm 0.213$ & $F=0.392, p=0.553$ \\
\hline Left middle temporal gyrus & $-62,-17,-7$ & $-0.0071 \pm 0.199$ & $-0.024 \pm 0.218$ & $F=0.187, p=0.667$ \\
\hline Right parietal white matter & $+32,-27,+33$ & $-0.147 \pm 0.147$ & $-0.141 \pm 0.129$ & $F=0.073, p=0.788$ \\
\hline Right middle parietal gyrus & $+59,-42,+9$ & $-0.117 \pm 0.25$ & $-0.141 \pm 0.25$ & $F=0.274, p=0.602$ \\
\hline Right precuneus & $+8,-58,+14$ & $0.019 \pm 0.21$ & $-0.039 \pm 0.189$ & $F=2.504, p=0.116$ \\
\hline Right cerebellum & $+41,-67,-22$ & $-0.056 \pm 0.243$ & $-0.121 \pm 0.24$ & $F=2.154, p=0.145$ \\
\hline Right prefrontal white matter & $+18,+38,+1$ & $-0.08 \pm 0.15$ & $-0.047 \pm 0.156$ & $F=1.483, p=0.226$ \\
\hline Right frontal white matter & $+18,-12,+42$ & $-0.019 \pm 0.146$ & $-0.028 \pm 0.164$ & $F=0.077, p=0.782$ \\
\hline Left frontal pole & $-20,+62,-9$ & $-0.193 \pm 0.266$ & $-0.0396 \pm 0.158$ & $F=14.854, p<0.001$ \\
\hline Left anterior cingulate & $-11,+45,+17$ & $-0.046 \pm 0.255$ & $-0.086 \pm 0.228$ & $F=0.848, p=0.359$ \\
\hline
\end{tabular}

The cluster in the ventral medial PFC (MNI coordinates: $+0,+23,-24)$ was omitted from these analyses because it was located on the midline.

being related to poor cognitive functioning. Future longitudinal research is needed to confirm this idea.

Of note, there are aspects of the study design that potentially limit the interpretation of results. Our data are based on a single scan obtained at one point in development. It is possible that brain development is just delayed in children who were subjected to high levels of cumulative life stress. The major focus of investigation, the PFC, is still developing during the developmental stage when the MRI scans were obtained. The brains of children who have experienced greater amounts of life stress could "equalize" over time. The trajectory of brain development has proven to be a robust predictor of cognitive functions during childhood and adolescence (Shaw et al., 2006). In addition, later environmental experience could aid in reducing any differences. Environment enrichment and exercise, for example, have both been linked to increases in neurogenesis in the brain (Kempermann et al., 1997; van Praag et al., 1999, 2005) and even recovery after a lesion (Will et al., 1977). Future work will attempt to investigate these ideas by examining longitudinal changes in brain structure and function.

We initially hypothesized that prefrontal circuitry would be uniquely affected by cumulative life stress. However, we discovered brain regions in addition to the PFC that were related to cumulative life stress (Table 2). Many of these areas were related to sensory processing (e.g., occipital regions) and multisensory integration (i.e., parietal white matter, precuneus) brain regions. An emerging body of research in adults exposed to traumatic experiences during childhood is in line with these findings. Lower gray matter volumes and white matter fiber integrity in the visual cortex have been noted in adults who witnessed domestic violence or who were sexually abused in childhood (Tomoda et al., 2009; Choi et al., 2012). Such results also fit with nonhuman models of prenatal stress (Schneider et al., 2008) that have found alterations in sensory processing in rhesus macaques stressed early in development compared with those with no stress exposure. Future research is needed to clarify brain-behavior associations related to stress exposure and sensory processing and integration. Stress may affect these regions through excitotoxic events similar to the hippocampus, especially during development when these regions are still developing greatly. Changes in these brain areas could have large effects on a wide range of behaviors, including the control of attention, the regulation of emotions, and memory formation.

In the regions not initially hypothesized, only two specific areas were related to cumulative life stress and also behavior (Ta- ble 6): the precuneus and the middle temporal gyrus. These results fit very well with studies examining the effects of acute stress on brain and behavior in humans. Previous research has found that acute stress in humans disrupted functional brain activity and connectivity in frontoparietal networks (Dedovic et al., 2009; Liston et al., 2009). Novel perfusion functional MRI research examining cerebral blood flow during acute psychological stress found associations between blood flow and cortisol in the anterior cingulate, precuneus, and portions of temporal cortex (Wang et al. 2005). When we used multiple regression models to predict SWM performance, the PFC clusters were the only brain regions contributing significant variance, along with cumulative life stress, pubertal stage, and acute stressors. The precuneus was nearly significant, underscoring the need for strong focus on this region in future research examining stress-induced decrements in cognitive functioning.

Previous research suggested that stress-induced neurobiological changes may exhibit asymmetries in effects. Work by Sullivan and Gratton (1998), for example, found asymmetric mesocortical dopamine activation dependent on the type of stress and that regulation of dopamine responses to multiple types of stress was tightly coupled in the right hemisphere. Such findings suggest a specialized role for right cortical mechanisms in the integration of emotional and physiological responses to stressful situations. In our sample, we found significant asymmetrical effects for the left frontal pole $(p<0.001)$, along with portions of the right $(p<$ $0.001)$ and left $(p=0.009)$ occipital lobe. These regions, however, were not strongly related to behavior measured in this study (i.e., SWM). Of important note, anatomical variability means that the left- and right-sided clusters are not necessarily in homologous locations despite the mirroring of clusters. However, despite this limitation, such an analysis does provide some useful information that would not be available without it. In particular, the analysis can reveal that a cluster on one side is significantly different from a homologous cluster. This has important added value. Nonhuman animal models or research in adult humans using positron emission tomography could clarify this possible stress-related asymmetry.

This study is one of the first to link cumulative life stress in childhood and adolescence to differences in PFC and also to find that these structural alterations are associated with executive functioning. We found alterations in prefrontal gray matter (near the frontal poles) and white matter (near the forceps minor). Previous research suggests that gray matter in this region is in- 
volved with strategic processes in memory retrieval, such as maintaining a pending state for subsequent retrieval and execution after completion of the ongoing one (Koechlin and Hyafil, 2007). For the white matter results, these brain associations were found in a portion of tissue that connects the lateral and medial surfaces of the frontal lobes. Previous research with diffusion tensor imaging has found associations when looking at fractional anisotropy and cognitive functioning in certain clinical populations, with lower fractional anisotropy being related to poorer cognitive functioning (Van Hecke et al., 2010). Both of these ideas are consistent with our results.

By specifically linking cumulative life stress to focal neuroanatomical alterations and linking those alterations to behavioral performance, our results suggest that structural differences in the PFC may serve as one mechanism through which greater cumulative life stress engenders poorer executive functioning. The PFC is central to attention, working memory, cognitive control, and emotion regulation processes, with damage to this region leading to impairments in planning, goal attainment, problem-solving ability, and the regulation of emotion (Stuss and Levine, 2002; Braver et al., 2010). Structural stress-induced changes in this region may lead to impairments in these processes, thereby undermining cognitive performance during development.

\section{References}

Arnsten AF (2009) Stress signalling pathways that impair prefrontal cortex structure and function. Nat Rev Neurosci 10:410-422.

Asato MR, Terwilliger R, Woo J, Luna B (2010) White matter development in adolescence: a DTI study. Cereb Cortex 20:2122-2131.

Avants B, Gee JC (2004) Geodesic estimation for large deformation anatomical shape averaging and interpolation. Neuroimage 23:S139-S150.

Baron RM, Kenny DA (1986) The moderator-mediator variable distinction in social psychological research: conceptual, strategic, and statistical considerations. J Pers Soc Psychol 51:1173-1182.

Boyce WT, Chesterman E (1990) Life events, social support, and cardiovascular reactivity in adolescence. J Dev Behav Pediatr 11:105-111.

Braver TS, Cole MW, Yarkoni T (2010) Vive les differences! Individual variation in neural mechanisms of executive control. Curr Opin Neurobiol 20:242-250.

Brickman AM, Zimmerman ME, Paul RH, Grieve SM, Tate DF, Cohen RA, Williams LM, Clark CR, Gordon E (2006) Regional white matter and neuropsychological functioning across the adult lifespan. Biol Psychiatry 60:444-453.

Choi J, Jeong B, Polcari A, Rohan ML, Teicher MH (2012) Reduced fractional anisotropy in the visual limbic pathway of young adults witnessing domestic violence in childhood. Neuroimage 59:1071-1079.

Cook SC, Wellman CL (2004) Chronic stress alters dendritic morphology in rat medial prefrontal cortex. J Neurobiol 60:236-248.

Dedovic K, D'Aguiar C, Pruessner JC (2009) What stress does to your brain: a review of neuroimaging studies. Can J Psychiatry 54:6-15.

D’Esposito M, Detre JA, Alsop DC, Shin RK, Atlas S, Grossman M (1995) The neural basis of the central executive system of working memory. Nature 378:279-281.

Dias R, Robbins TW, Roberts AC (1996) Dissociation in prefrontal cortex of affective and attentional shifts. Nature 380:69-72.

Dienes KA, Hammen C, Henry RM, Cohen AN, Daley SE (2006) The stress sensitization hypothesis: understanding the course of bipolar disorder. J Affect Disord 95: 43-49.

Ellis BJ (2004) Timing of pubertal maturation in girls: an integrated life history approach. Psychol Bull 130:920-958.

Evans GW, Schamberg MA (2009) Childhood poverty, chronic stress, and adult working memory. Proc Natl Acad Sci U S A 106:6545-6549.

Gunning-Dixon FM, Raz N (2003) Neuroanatomical correlates of selected executive functions in middle-aged and older adults: a prospective MRI study. Neuropsychologia 41:1929-1941.

Guyer AE, Choate VR, Pine DS, Nelson EE (2012) Neural circuitry underlying affective response to peer feedback in adolescence. Soc Cogn Affect Neurosci 7:81-92.

Hanson JL, Chung MK, Avants BB, Shirtcliff EA, Gee JC, Davidson RJ, Pollak
SD (2010) Early stress is associated with alterations in the orbitofrontal cortex: a tensor-based morphometry investigation of brain structure and behavioral risk. J Neurosci 30:7466-7472.

Haveman R, Wolfe B (1995) The determinants of children's attainments: a review of methods and findings. J Econ Lit 33:1829-1878.

Hazel NA, Hammen C, Brennan PA, Najman J (2008) Early childhood adversity and adolescent depression: the mediating role of continued stress. Psychol Med 38:581-589.

Holmes A, Wellman CL (2009) Stress-induced prefrontal reorganization and executive dysfunction in rodents. Neurosci Biobehav Rev 33: 773-783.

Kempermann G, Kuhn HG, Gage FH (1997) More hippocampal neurons in adult mice living in an enriched environment. Nature 386:493-495.

Klein A, Andersson J, Ardekani BA, Ashburner J, Avants B, Chiang MC, Christensen GE, Collins DL, Gee J, Hellier P, Song JH, Jenkinson M, Lepage C, Rueckert D, Thompson P, Vercauteren T, Woods RP, Mann JJ, Parsey RV (2009) Evaluation of 14 nonlinear deformation algorithms applied to human brain MRI registration. Neuroimage 46:786-802.

Koechlin E, Hyafil A (2007) Anterior prefrontal function and the limits of human decision-making. Science 318:594-598.

Lenroot RK, Giedd JN (2006) Brain development in children and adolescents: insights from anatomical magnetic resonance imaging. Neurosci Biobehav Rev 30:718-729.

Liston C, Miller MM, Goldwater DS, Radley JJ, Rocher AB, Hof PR, Morrison JH, McEwen BS (2006) Stress-induced alterations in prefrontal cortical dendritic morphology predict selective impairments in perceptual attentional set-shifting. J Neurosci 26:7870-7874.

Liston C, McEwen BS, Casey BJ (2009) Psychosocial stress reversibly disrupts prefrontal processing and attentional control. Proc Natl Acad Sci U S A 106:912-917.

Luciana M, Nelson CA (2002) Assessment of neuropsychological function through use of the Cambridge Neuropsychological Testing Automated Battery: performance in 4- to 12-year-old children. Dev Neuropsychol 22:595-624.

Lupien SJ, McEwen BS, Gunnar MR, Heim C (2009) Effects of stress throughout the lifespan on the brain, behaviour and cognition. Nat Rev Neurosci 10:434-445.

Lyons DM, Afarian H, Schatzberg AF, Sawyer-Glover A, Moseley ME (2002) Experience-dependent asymmetric variation in primate prefrontal morphology. Behav Brain Res 136:51-59.

Monroe SM (2008) Modern approaches to conceptualizing and measuring human life stress. Annu Rev Clin Psychol 4:33-52.

Murphy BL, Arnsten AF, Jentsch JD, Roth RH (1996) Dopamine and spatial working memory in rats and monkeys: pharmacological reversal of stressinduced impairment. J Neurosci 16:7768-7775.

Oei NY, Everaerd WT, Elzinga BM, van Well S, Bermond B (2006) Psychosocial stress impairs working memory at high loads: an association with cortisol levels and memory retrieval. Stress 9:133-141.

Owen AM, Morris RG, Sahakian BJ, Polkey CE, Robbins TW (1996) Double dissociations of memory and executive functions in working memory tasks following frontal lobe excisions, temporal lobe excisions or amygdalo-hippocampectomy in man. Brain 119:1597-1615.

Parker KJ, Buckmaster CL, Justus KR, Schatzberg AF, Lyons DM (2005) Mild early life stress enhances prefrontal-dependent response inhibition in monkeys. Biol Psychiatry 57:848-855.

Preacher KJ, Hayes AF (2004) SPSS and SAS procedures for estimating indirect effects in simple mediation models. Behav Res Methods Instrum Comput 36:717-731.

Radley JJ, Rocher AB, Miller M, Janssen WG, Liston C, Hof PR, McEwen BS, Morrison JH (2006) Repeated stress induces dendritic spine loss in the rat medial prefrontal cortex. Cereb Cortex 16:313-320.

Reich W (2000) Diagnostic interview for children and adolescents (DICA). J Am Acad Child Adolesc Psychiatry 39:59-66.

Rudolph KD, Flynn M (2007) Childhood adversity and youth depression: influence of gender and pubertal status. Dev Psychopathol 19:497-521.

Rudolph KD, Hammen C (1999) Age and gender as determinants of stress exposure, generation, and reactions in youngsters: a transactional perspective. Child Dev 70:660-677.

Sánchez MM, Hearn EF, Do D, Rilling JK, Herndon JG (1998) Differential rearing affects corpus callosum size and cognitive function of rhesus monkeys. Brain Res 812:38-49.

Schneider ML, Moore CF, Gajewski LL, Larson JA, Roberts AD, Converse AK, 
DeJesus OT (2008) Sensory processing disorder in a primate model: evidence from a longitudinal study of prenatal alcohol and prenatal stress effects. Child Dev 79:100-113.

Schwabe L, Wolf OT, Oitzl MS (2010) Memory formation under stress: quantity and quality. Neurosci Biobehav Rev 34:584-591.

Schwabe L, Joëls M, Roozendaal B, Wolf OT, Oitzl MS (2011) Stress effects on memory: an update and integration. Neurosci Biobehav Rev. Advance online publication. Retrieved April 26, 2012. doi:10.1016/j.neubiorev.2011.07.002.

Shaw P, Greenstein D, Lerch J, Clasen L, Lenroot R, Gogtay N, Evans A, Rapoport J, Giedd J (2006) Intellectual ability and cortical development in children and adolescents. Nature 440:676-679.

Shirtcliff EA, Dahl RE, Pollak SD (2009) Pubertal development: correspondence between hormonal and physical development. Child Dev 80:327-337.

Sobel ME (1986) Some new results on indirect effects and their standard errors in covariance structure analysis. In: Sociological methodology (Tuma N, ed), pp 159-186. Washington, DC: American Sociological Association.

Sowell ER, Delis D, Stiles J, Jernigan TL (2001) Improved memory functioning and frontal lobe maturation between childhood and adolescence: a structural MRI study. J Int Neuropsychol Soc 7:312-322.

Straus MA, Hamby SL, Finkelhor D, Moore DW, Runyan D (1998) Identification of child maltreatment with the parent-child conflict tactics scales: development and psychometric data for a national sample of American parents. Child Abuse Negl 22:249-270.

Stuss DT, Levine B (2002) Adult clinical neuropsychology: lessons from studies of the frontal lobes. Annu Rev Psychol 53:401-433.

Tang AC, Akers KG, Reeb BC, Romeo RD, McEwen BS (2006) Programming social, cognitive, and neuroendocrine development by early exposure to novelty. Proc Natl Acad Sci U S A 103:15716-15721.

Tang AC, Reeb-Sutherland BC, Yang Z, Romeo RD, McEwen BS (2011) Neonatal novelty-induced persistent enhancement in offspring spatial memory and the modulatory role of maternal self-stress regulation. J Neurosci 31:5348-5352.

Tisserand DJ, van Boxtel MP, Pruessner JC, Hofman P, Evans AC, Jolles J (2004) A voxel-based morphometric study to determine individual differences in gray matter density associated with age and cognitive change over time. Cereb Cortex 14:966-973.

Tomoda A, Navalta CP, Polcari A, Sadato N, Teicher MH (2009) Childhood sexual abuse is associated with reduced gray matter volume in visual cortex of young women. Biol Psychiatry 66:642-648.

Van Hecke W, Nagels G, Leemans A, Vandervliet E, Sijbers J, Parizel PM (2010) Correlation of cognitive dysfunction and diffusion tensor MRI measures in patients with mild and moderate multiple sclerosis. J Magn Reson Imaging 31:1492-1498.

van Praag H, Shubert T, Zhao C, Gage FH (2005) Exercise enhances learning and hippocampal neurogenesis in aged mice. J Neurosci 25:8680-8685.

van Praag H, Kempermann G, Gage FH (1999) Running increases cell proliferation and neurogenesis in the adult mouse dentate gyrus. Nat Neurosci 2:266-270.

Waldfogel J, Han WJ, Brooks-Gunn J (2002) The effects of early maternal employment on child cognitive development. Demography 39:369-392.

Wethington E, Brown G, Kessler R (1995) Interview measurement of stressful life events. In: Measuring stress (Cohen S, Kessler R, Underwood GL, ed), pp 59-79. New York: Oxford UP.

Will BE, Rosenzweig MR, Bennett EL, Hebert M, Morimoto H (1977) Relatively brief environmental enrichment aids recovery of learning capacity and alters brain measures after postweaning brain lesions in rats. J Comp Physiol Psychol 91:33-50.

Williams GV, Goldman-Rakic PS (1995) Modulation of memory fields by dopamine D1 receptors in prefrontal cortex. Nature 376:572-575.

Worsley KJ, Liao CH, Aston J, Petre V, Duncan GH, Morales F, Evans AC (2002) A general statistical analysis for fMRI data. Neuroimage 15:1-15.

Zelazo PD, Craik FI, Booth L (2004) Executive function across the life span. Acta Psychologica 115:167-183. 